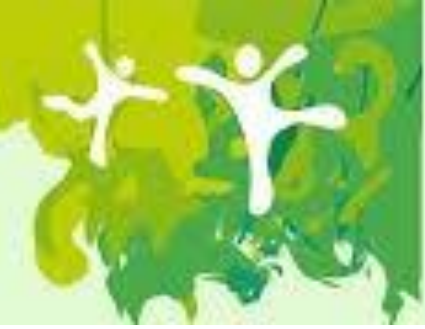

\title{
COMPOSIÇAO CORPORAL DE CRIANÇAS COM ADRENARCA PRECOCE IDIOPATICA EM UM MUNICIPIO DE SAO CARLOS- AP, BRASIL
}

\section{Pôster}

Autores deste trabalho:

Vanessa de Oliveira Furino: Departamento de Enfermagem- Universidade Federal de São Carlos (UFSCar)

Fernanda de Oliveira Furino: Departamento de Enfermagem- Universidade Federal de São Carlos (UFSCar)

Lucimar Retto da Silva de Avó: Departamento de Medicina -Universidade Federal de São Carlos (UFSCar)

Lucila Leico Kagohara Elias: Departamento de Fisiologia -Universidade de São Paulo (USP)

Debora Gusmão Melo: Departamento de Medicina -Universidade Federal de São Carlos (UFSCar)

Carla Maria Ramos Germano : Departamento de Medicina -Universidade Federal de São Carlos (UFSCar)

Área do Trabalho: Enfermagem pediátrica

Número de inscrição: 5247

Data da submissão:05/08/2016 às 16:55

\section{Justificativa}

O surgimento prematuro de pelos pubianos e/ou axilares, acne e odor corporal antes dos 8 anos de idade em meninas e antes dos 9 em meninos caracteriza a situação clínica adrenarca precoce. As repercussões metabólicas incluem risco aumentado de desenvolver síndrome metabólica (SM), diabetes mellitus tipo 2 (DM2), dislipidemia, além de maior predisposição a síndrome de ovário policísticos na vida adulta.

\section{Objetivo(s)}

Analisar parâmetros antropométricos e metabólicos em crianças com diagnóstico de AP.

\section{Método(s)}

Estudo quantitativo transversal exploratório, no qual foram avaliadas 15 crianças com diagnóstico de AP e 15 controles (C) pareados, recrutados na rede pública de saúde da 
cidade de São Carlos, SP. Foram determinados dados antropométricos: altura, peso, circunferência abdominal (CA), prega cutânea tricipital, pressão arterial sistólica e pressão arterial diastólica. Os participantes realizaram exames de glicemia, insulina, colesterol total, LDL, HDL, triglicerídeos (TG), DHEA-S e 17OHP. A análise dos dados foi realizada através do programa GraphPad Prism 5. O teste de Wilcoxon pareado foi utilizado para avaliar a diferença entre as médias.

\section{Resultado(s)}

Não houve diferenças significativas entre os parâmetros antropométricos entre os grupos C e AP. Os valores de TG $(99 \pm 14,8 \mathrm{mg} / \mathrm{dl}$ nos indivíduos $\mathrm{C}$ versus $68 \pm 9,1 \mathrm{mg} / \mathrm{dl}$ nos indivíduos com AP) $(\mathrm{p}=0,0233)$ foram significativamente diferentes entre os grupos. $\mathrm{A}$ categorização dos grupos em relação à circunferência abdominal (CA) evidenciou que $6,7 \%$ do grupo $\mathrm{C}$ versus 33,3\% do grupo AP tinha $\mathrm{CA}$ acima do p90. Adicionalmente, $6,7 \%$ do grupo $C$ versus $20 \%$ do grupo AP apresentava relação $\mathrm{G} / \mathrm{I}<7$.

\section{Conclusão(ões)}

Este estudo evidenciou maior porcentagem de indivíduos com hiperinsulinemia, resistência insulínica e CA elevada no grupo AP, importantes marcadores de SM e doenças cardiovasculares futuras. Ainda que diferenças não foram evidenciadas na maioria dos parâmetros antropométricos e metabólicos é possível que essas alterações não sejam evidentes em idade peripuberal, mas que se desenvolvam com o avanço da idade cronológica e desenvolvimento da puberdade. Nesse caso, o acompanhamento das crianças desse estudo se faz necessário, de forma a detectar precocemente impactos negativos à saúde ao longo do crescimento. 